\title{
A NEW EXAMPLE OF A MINIMAL NONFINITELY BASED SEMIGROUP
}

\author{
WEN TING ZHANG ${ }^{\bowtie}$ and YAN FENG LUO
}

(Received 3 April 2011)

\section{Abstract}

Only three of the 15973 distinct six-element semigroups are presently known to be nonfinitely based. This paper introduces a fourth example.

2010 Mathematics subject classification: primary 20M07.

Keywords and phrases: semigroups, Lee's semigroup, finitely based.

\section{Introduction}

A semigroup is finitely based if its identities are finitely axiomatizable. In the 1960s, Perkins proved that the six-element Brandt monoid

$$
B_{2}^{1}=\left\langle a, b, 1 \mid a^{2}=b^{2}=0, a b a=a, b a b=b\right\rangle
$$

is nonfinitely based as a semigroup [8]. In the decades that followed, two more similar semigroups were discovered: the six-element monoid

$$
A_{2}^{1}=\left\langle a, b, 1 \mid a^{2}=a b a=a, b a b=b, b^{2}=0\right\rangle
$$

was independently shown by Sapir [10] and Trahtman [12] to be nonfinitely based, and the nonfinite basis property of the six-element semigroup

$$
A_{2}^{g}=\left\langle\begin{array}{l|l}
a, b, g, z & \begin{array}{l}
a^{2}=a b a=a, a g=g a=b g=g b=z g=g z=g, \\
b a b=b, a z=z a=b z=z b=b^{2}=g^{2}=z^{2}=z
\end{array}
\end{array}\right\}
$$

follows from results of either Mashevitskiŭ [7] or Volkov [14] (an explicit basis for $A_{2}^{g}$ was recently given by Lee and Volkov [6]). Since all semigroups with five or fewer elements are finitely based $[3,13]$, the semigroups $A_{2}^{1}, A_{2}^{g}$, and $B_{2}^{1}$ are minimal with respect to being nonfinitely based. Apart from these three examples, no other minimal nonfinitely based semigroup is known. This prompted Lee and Li [4] to ask the following fundamental question: is there any minimal nonfinitely based semigroup that is distinct from $A_{2}^{1}, A_{2}^{g}$, and $B_{2}^{1}$ ? Recall that two semigroups are distinct if they 
are neither isomorphic nor anti-isomorphic. There are 15973 distinct six-element semigroups [9], among which 1373 are monoids [2] and 14600 are nonunital.

Lee and Li partially answered the above question by proving that among all distinct six-element monoids, only $A_{2}^{1}$ and $B_{2}^{1}$ are nonfinitely based [4]. After establishing this result, Lee continued investigating the finite basis problem for the remaining 14600 nonunital six-element semigroups. In a private communication, he suggested that the authors examine the finite basis problem of several six-element semigroups. It turned out that one of these semigroups,

$$
L=\left\langle d, e \mid d^{2}=d, e^{2}=e, d e d=0\right\rangle,
$$

is nonfinitely based. The main aim of this paper is to present a proof of this result. Consequently, the number of distinct minimal nonfinitely based semigroups is at least four.

This paper is organized as follows. In Section 3, it is shown that Lee's semigroup $L$ has a basis that consists of identities with some special property. In Section 4 , some restrictions on the identities satisfied by $L$ are established. The semigroup $L$ is then shown to be nonfinitely based in Section 5 .

Recall that a semigroup $S$ is inherently nonfinitely based if any locally finite variety containing $S$ is nonfinitely based. Sapir proved that the semigroups $A_{2}^{1}$ and $B_{2}^{1}$ are inherently nonfinitely based [10]. On the other hand, a semigroup that satisfies a nontrivial identity of the form $x y x \approx \mathbf{w}$ is not inherently nonfinitely based [10]. Since the semigroups $A_{2}^{g}$ and $L$ satisfy the identities $x y x \approx x y x y x$ and $x y x \approx x y x^{2}$ respectively, they are not inherently nonfinitely based.

\section{Preliminaries}

Let $\mathcal{X}$ be a countably infinite alphabet throughout. For any subset $\mathcal{Y}$ of $\mathcal{X}$, let $\mathcal{Y}^{+}$ and $\mathcal{Y}^{*}$ denote the free semigroup and free monoid over $\mathcal{Y}$ respectively. Elements of $\mathcal{X}$ are called letters and elements of $\mathcal{X}^{*}$ are called words. A word is a singleton if it is a single letter. For any word w:

- $\quad$ the head of $\mathbf{w}$, denoted by $\mathrm{h}(\mathbf{w})$, is the first letter occurring in $\mathbf{w}$;

- the tail of $\mathbf{w}$, denoted by $\mathrm{t}(\mathbf{w})$, is the last letter occurring in $\mathbf{w}$;

- the content of $\mathbf{w}$, denoted by $\operatorname{con}(\mathbf{w})$, is the set of letters occurring in $\mathbf{w}$;

- $\quad$ the number of occurrences of a letter $x$ in $\mathbf{w}$ is denoted by $\operatorname{occ}(x, \mathbf{w})$;

- $\quad$ a letter $x$ is simple in $\mathbf{w}$ if $\operatorname{occ}(x, \mathbf{w})=1$;

- $\quad$ the set of simple letters of $\mathbf{w}$ is denoted by $\operatorname{sim}(\mathbf{w})$;

- $\quad$ the set of nonsimple letters of $\mathbf{w}$ is denoted by non(w).

Note that $\operatorname{con}(\mathbf{w})=\operatorname{sim}(\mathbf{w}) \cup \operatorname{non}(\mathbf{w})$ and $\operatorname{sim}(\mathbf{w}) \cap \operatorname{non}(\mathbf{w})=\emptyset$. Two words $\mathbf{u}$ and $\mathbf{v}$ are disjoint if $\operatorname{con}(\mathbf{u}) \cap \operatorname{con}(\mathbf{v})=\emptyset$.

An identity is written as $\mathbf{u} \approx \mathbf{v}$ where $\mathbf{u}, \mathbf{v} \in \mathcal{X}^{+}$. An identity $\mathbf{u} \approx \mathbf{v}$ is nontrivial if $\mathbf{u} \neq \mathbf{v}$. A semigroup $S$ satisfies an identity $\mathbf{u} \approx \mathbf{v}$ if for any substitution $\varphi$ from $\mathcal{X}$ into $S$, the elements $\mathbf{u} \varphi$ and $\mathbf{v} \varphi$ of $S$ are equal. A set $\Sigma$ of identities satisfied by a semigroup $S$ is a basis for $S$ if every identity of $S$ can be deduced from $\Sigma$. A semigroup is finitely 
based if it has a finite basis. For any concept of universal algebra that appears but is not defined in this paper, refer to Burris and Sankappanavar [1] and Shevrin and Volkov [11].

By writing $a=e d e, b=d e$, and $c=e d$ in the semigroup $L$, the multiplication table for $L$ is given by

\begin{tabular}{l|llllll}
$L$ & 0 & $a$ & $b$ & $c$ & $d$ & $e$ \\
\hline 0 & 0 & 0 & 0 & 0 & 0 & 0 \\
$a$ & 0 & 0 & 0 & 0 & 0 & $a$ \\
$b$ & 0 & 0 & 0 & 0 & 0 & $b$ \\
$c$ & 0 & 0 & $a$ & 0 & $c$ & $a$ \\
$d$ & 0 & 0 & $b$ & 0 & $d$ & $b$ \\
$e$ & 0 & $a$ & $a$ & $c$ & $c$ & $e$
\end{tabular}

Lemma 1. Suppose that the semigroup L satisfies an identity $\mathbf{u} \approx \mathbf{v}$. Then $\operatorname{sim}(\mathbf{u})=$ $\operatorname{sim}(\mathbf{v})$ and $\operatorname{non}(\mathbf{u})=\operatorname{non}(\mathbf{v})$.

Proof. Suppose that $x \in \operatorname{con}(\mathbf{u})$ and $x \notin \operatorname{con}(\mathbf{v})$. Then letting $\varphi: \mathcal{X} \rightarrow L$ be the substitution that maps $x$ to 0 and any other letter to $e$, we obtain the contradiction $\mathbf{u} \varphi=0 \neq e=\mathbf{v} \varphi$. Thus $\operatorname{con}(\mathbf{u})=\operatorname{con}(\mathbf{v})$. Suppose that $x \in \operatorname{sim}(\mathbf{u})$ and $x \notin \operatorname{sim}(\mathbf{v})$. Then letting $\psi: \mathcal{X} \rightarrow L$ be the substitution that maps $x$ to $a$ and any other letter to $e$, we obtain the contradiction $\mathbf{u} \psi=a \neq 0=\mathbf{v} \psi$. Therefore $\operatorname{sim}(\mathbf{u})=\operatorname{sim}(\mathbf{v})$, whence $\operatorname{non}(\mathbf{u})=\operatorname{non}(\mathbf{v})$.

\section{Connected identities}

A semigroup $S$ is idempotent-separable if the following holds for any distinct elements $x$ and $y$ of $S$ : $e x \neq e y$ for some idempotent $e$ in $S$, and $x f \neq y f$ for some idempotent $f$ in $S$. (The idempotents $e$ and $f$ need not be distinct.) It is easy to verify that the semigroup $L$ is idempotent-separable by referring to its multiplication table in Section 2.

The variety generated by a semigroup $S$ is the class of all semigroups that satisfy all identities of $S$. The four-element semigroup

$$
A_{0}=\left\langle a, b \mid a^{2}=a, b^{2}=b, a b=0\right\rangle
$$

is required in the following result.

Lemma 2 [5, Proposition 3.2]. Let $S$ be any idempotent-separable semigroup such that the variety generated by $S$ contains the semigroup $A_{0}$. Suppose that $S$ satisfies an identity $\mathbf{u} \approx \mathbf{v}$ where the word $\mathbf{u}$ can be written as $\mathbf{u}=\mathbf{u}^{\prime} \mathbf{u}^{\prime \prime}$ for some disjoint words $\mathbf{u}^{\prime}, \mathbf{u}^{\prime \prime} \in \mathcal{X}^{+}$. Then the word $\mathbf{v}$ can be written as $\mathbf{v}=\mathbf{v}^{\prime} \mathbf{v}^{\prime \prime}$ for some disjoint words $\mathbf{v}^{\prime}, \mathbf{v}^{\prime \prime} \in \mathcal{X}^{+}$such that $\operatorname{con}\left(\mathbf{u}^{\prime}\right)=\operatorname{con}\left(\mathbf{v}^{\prime}\right)$ and $\operatorname{con}\left(\mathbf{u}^{\prime \prime}\right)=\operatorname{con}\left(\mathbf{v}^{\prime \prime}\right)$. Further, $S$ satisfies the identities $\mathbf{u}^{\prime} \approx \mathbf{v}^{\prime}$ and $\mathbf{u}^{\prime \prime} \approx \mathbf{v}^{\prime \prime}$.

A word of length at least two is connected if it cannot be written as a product of two disjoint nonempty words. A connected identity is an identity that is formed by a pair of connected words. 
Lemma 3. The semigroup L has a basis that contains only connected identities.

Proof. By identifying the elements $a$ and $b$ of $L$ with 0 , we obtain a semigroup isomorphic to $A_{0}$. Hence the semigroup $A_{0}$ belongs to the variety generated by $L$. As observed earlier, the semigroup $L$ is idempotent-separable, whence Lemma 2 is applicable to $L$.

Let $\mathbf{u} \approx \mathbf{v}$ be any nontrivial identity satisfied by the semigroup $L$. Write $\mathbf{u}=$ $\mathbf{u}_{1} \cdots \mathbf{u}_{m}$ where $\mathbf{u}_{1}, \ldots, \mathbf{u}_{m} \in \mathcal{X}^{+}$are pairwise disjoint words, each of which is either connected or a singleton. By repeatedly applying Lemma 2, we obtain $\mathbf{v}=\mathbf{v}_{1} \cdots \mathbf{v}_{m}$ for some pairwise disjoint words $\mathbf{v}_{1}, \ldots, \mathbf{v}_{m} \in \mathcal{X}^{+}$such that for each $i$, the semigroup $L$ satisfies the identity $\mathbf{u}_{i} \approx \mathbf{v}_{i}$ with $\operatorname{con}\left(\mathbf{u}_{i}\right)=\operatorname{con}\left(\mathbf{v}_{i}\right)$. For any $i$, it follows from Lemma 1 that if either $\mathbf{u}_{i}$ or $\mathbf{v}_{i}$ is a singleton, then they must be the same word, whence the identity $\mathbf{u}_{i} \approx \mathbf{v}_{i}$ is trivial.

Now suppose that, for some $i$, both $\mathbf{u}_{i}$ and $\mathbf{v}_{i}$ are not singletons and $\mathbf{v}_{i}$ is not connected. Then $\mathbf{v}_{i}=\mathbf{v}_{i}^{\prime} \mathbf{v}_{i}^{\prime \prime}$ for some disjoint words $\mathbf{v}_{i}^{\prime}, \mathbf{v}_{i}^{\prime \prime} \in \mathcal{X}^{+}$. But then Lemma 2 implies that $\mathbf{u}_{i}=\mathbf{u}_{i}^{\prime} \mathbf{u}_{i}^{\prime \prime}$ for some disjoint words $\mathbf{u}_{i}^{\prime}, \mathbf{u}_{i}^{\prime \prime} \in \mathcal{X}^{+}$, contradicting the connectedness of $\mathbf{u}_{i}$. Hence $\mathbf{v}_{i}$ must be connected.

We have just shown that the semigroup $L$ satisfies the nontrivial identity $\mathbf{u} \approx \mathbf{v}$ if and only if it satisfies the identities $\mathbf{u}_{1} \approx \mathbf{v}_{1}, \ldots, \mathbf{u}_{m} \approx \mathbf{v}_{m}$; each of these $m$ identities is either trivial or connected. Consequently, any nontrivial identity in a basis for $L$ that is not connected can be replaced by identities that are connected.

\section{Identities satisfied by $L$}

It is routine to verify that the semigroup $L$ satisfies the identities

$$
x^{3} \approx x^{2}, \quad x^{2} y x \approx x y x, \quad x y x^{2} \approx x y x .
$$

In this section, we present some restrictions on identities satisfied by $L$.

LeMma 4. The semigroup L does not satisfy the identity

$$
x y^{2} x \approx \mathbf{z}
$$

for any $\mathbf{z} \in\left\{x y x y x, x y x y, y x y x, x^{2} y^{2}, y^{2} x^{2}\right\}$.

Proof. Let $x=e$ and $y=d$. Then the left-hand side of (2) is $e d^{2} e=a$. But the righthand side of (2) can only be edede $=e d e d=$ dede $=0, e^{2} d^{2}=c$, or $d^{2} e^{2}=b$.

For each $n \geq 2$, define the sets of words

$$
\begin{aligned}
& \mathcal{P}_{n}=\left\{x^{r_{1}} y_{1}^{s_{1}} \cdots y_{n}^{s_{n}} x^{r_{2}} \mid r_{1}, r_{2} \geq 1, s_{1}, \ldots, s_{n} \geq 2\right\}, \\
& \mathcal{Q}_{n}=\left\{x^{r_{1}} y_{n}^{s_{n}} \cdots y_{1}^{s_{1}} x^{r_{2}} \mid r_{1}, r_{2} \geq 1, s_{1}, \ldots, s_{n} \geq 2\right\} .
\end{aligned}
$$

Note that any word in $\mathcal{P}_{n}$ written in reverse order belongs to $Q_{n}$, and vice versa.

Lemma 5. Let $\mathbf{u} \approx \mathbf{v}$ be any identity satisfied by the semigroup $L$ such that $\mathbf{u} \in \mathcal{P}_{n}$. Then $\mathbf{v} \in \mathcal{P}_{n} \cup Q_{n}$. 
Proof. By assumption,

$$
\mathbf{u}=x^{r_{1}} y_{1}^{s_{1}} \cdots y_{n}^{s_{n}} x^{r_{2}},
$$

for some $r_{1}, r_{2} \geq 1$ and $s_{1}, \ldots, s_{n} \geq 2$. By Lemma 1 , we have $\operatorname{sim}(\mathbf{v})=\operatorname{sim}(\mathbf{u})=\emptyset$ and $\operatorname{non}(\mathbf{v})=\operatorname{non}(\mathbf{u})=\left\{x, y_{1}, \ldots, y_{n}\right\}$. Hence

(a) $\operatorname{occ}(x, \mathbf{v})=k$ and $\operatorname{occ}\left(y_{i}, \mathbf{v}\right)=\ell_{i}$ for some $k, \ell_{i} \geq 2$.

Suppose that $\mathrm{h}(\mathbf{v})=y_{j}$ for some $j$. Then by letting $\varphi$ be the substitution $y_{i} \mapsto y^{2}$ for all $i$, we obtain

$$
x y^{2} x \stackrel{(1)}{\approx}(\mathbf{u} \varphi) x^{2} \underset{\approx}{\approx}(\mathbf{v} \varphi) x^{2} \in y^{2}\left\{x, y^{2}\right\}^{+} x^{2} \stackrel{(1)}{\approx}\left\{y^{2} x^{2}, y x y x\right\} .
$$

But this implies that the semigroup $L$ satisfies an identity from (2), contradicting Lemma 4 . Therefore $\mathrm{h}(\mathbf{v}) \neq y_{j}$ for any $j$, whence $\mathrm{h}(\mathbf{v})=x$. By a symmetrical argument, we have $\mathrm{t}(\mathbf{v})=x$. Hence $\mathbf{v}$ can be written as

(b) $\mathbf{v}=x^{k_{1}} \mathbf{w} x^{k_{2}}$ for some $k_{1}, k_{2} \geq 1$ and $\mathbf{w} \in\left\{x, y_{1}, \ldots y_{n}\right\}^{+}$with $\mathrm{h}(\mathbf{w}) \neq x \neq \mathrm{t}(\mathbf{w})$.

Suppose that $x \in \operatorname{con}(\mathbf{w})$, say $\mathbf{w}=\mathbf{w}_{1} x \mathbf{w}_{2}$ for some $\mathbf{w}_{1}, \mathbf{w}_{2} \in \mathcal{X}^{+}$. Then

$$
x y^{2} x \stackrel{(1)}{\approx} \mathbf{u} \varphi \stackrel{L}{\approx} \mathbf{v} \varphi \stackrel{(1)}{\approx} x y x y x .
$$

But this implies that the semigroup $L$ satisfies the identity $x y^{2} x \approx x y x y x$ from (2), contradicting Lemma 4. Therefore $x \notin \operatorname{con}(\mathbf{w})$, whence by (a) and (b) we have

(c) $\mathbf{v}=x^{k_{1}} \mathbf{w} x^{k_{2}}$ for some $\mathbf{w} \in\left\{y_{1}, \ldots y_{n}\right\}^{+}$and $k_{1}, k_{2} \geq 1$ with $k_{1}+k_{2}=k$.

Suppose that some $y_{i}$ in $\mathbf{w}$ is sandwiched between two occurrences of $y_{j}$ with $i \neq j$, say $\mathbf{w}=\mathbf{w}_{1} y_{j} \mathbf{w}_{2} y_{i} \mathbf{w}_{3} y_{j} \mathbf{w}_{4}$ for some $\mathbf{w}_{1}, \mathbf{w}_{2}, \mathbf{w}_{3}, \mathbf{w}_{4} \in X^{*}$. Then by letting $\chi$ be the substitution $z \mapsto x^{2}$ for all $z \neq y_{j}$, we obtain

$$
x y_{j}^{2} x \stackrel{(1)}{\approx} \mathbf{u} \chi \stackrel{L}{\approx} \mathbf{v} \chi \stackrel{(1)}{\approx} x y_{j} x y_{j} x .
$$

But this implies that the semigroup $L$ satisfies the identity $x y^{2} x \approx x y x y x$ from (2), contradicting Lemma 4. Hence no letter in $\mathbf{w}$ is sandwiched between two same occurrences of some other letter. It then follows from (a) and (c) that

(d) $\mathbf{v}=x^{k_{1}} y_{1 \pi}^{\ell_{1 \pi}} \cdots y_{n \pi}^{\ell_{n \pi}} x^{k_{2}}$ for some integers $k_{1}, k_{2} \geq 1$ and $\ell_{1 \pi}, \ldots, \ell_{n \pi} \geq 2$ and some permutation $\pi$ of $\{1, \ldots, n\}$.

Suppose that there exists some $i$ such that neither $y_{i}^{\ell_{i}} y_{i+1}^{\ell_{i+1}}$ nor $y_{i+1}^{\ell_{i+1}} y_{i}^{\ell_{i}}$ is a factor of $\mathbf{v}$. Then by letting $\psi$ be the substitution that maps both $y_{i}$ and $y_{i+1}$ to $y$ and any other letter to $x$, we obtain

$$
x y^{2} x \stackrel{(1)}{\approx} \mathbf{u} \psi \stackrel{L}{\approx} \mathbf{v} \psi \stackrel{(1)}{\approx} x y x y x .
$$

But this implies that the semigroup $L$ satisfies the identity $x y^{2} x \approx x y x y x$ from (2), contradicting Lemma 4. Therefore

(e) for each $i$, either $y_{i}^{\ell_{i}} y_{i+1}^{\ell_{i+1}}$ or $y_{i+1}^{\ell_{i+1}} y_{i}^{\ell_{i}}$ is a factor of $\mathbf{v}$.

It is now easy to show by (d) and (e) that either $\pi$ is a trivial permutation or $(1 \pi, 2 \pi, \ldots, n \pi)=(n, n-1, \ldots, 1)$, whence either $\mathbf{v} \in \mathcal{P}_{n}$ or $\mathbf{v} \in \mathcal{Q}_{n}$. 
Lemma 6. Let $\mathbf{u} \approx \mathbf{v}$ be any connected identity satisfied by the semigroup L such that $|\operatorname{con}(\mathbf{u})|,|\operatorname{con}(\mathbf{v})| \leq n$. Suppose that there exist words $\mathbf{e}, \mathbf{f} \in \mathcal{X}^{*}$ and an endomorphism $\varphi$ of $\mathcal{X}^{+}$such that $\mathbf{e}(\mathbf{u} \varphi) \mathbf{f} \in \mathcal{P}_{n}$. Then $\mathbf{e}(\mathbf{v} \varphi) \mathbf{f} \in \mathcal{P}_{n}$.

Proof. By assumption, $\mathbf{e}(\mathbf{u} \varphi) \mathbf{f}=\mathbf{x}$ where

$$
\mathbf{x}=x^{r_{1}} y_{1}^{s_{1}} \cdots y_{n}^{s_{n}} x^{r_{2}}
$$

for some $r_{1}, r_{2} \geq 1$ and $s_{1}, \ldots, s_{n} \geq 2$. Since the word $\mathbf{u}$ is connected, the image $\mathbf{u} \varphi$ is a connected factor of $\mathbf{x}$. The connected factors of $\mathbf{x}$ are exhausted by the following:

(F1) the factors of $x^{r_{1}}, y_{1}^{s_{1}}, \ldots, y_{n}^{s_{n}}, x^{r_{2}}$ of length at least two;

(F2) $x^{q_{1}} y_{1}^{s_{1}} \cdots y_{n}^{s_{n}} x^{q_{2}}$ where $1 \leq q_{1} \leq r_{1}$ and $1 \leq q_{2} \leq r_{2}$.

Case 1. $\mathbf{u} \varphi$ belongs to (F1). It suffices to assume that $\mathbf{u} \varphi$ is a factor of some $y_{i}^{s_{i}}$, since the argument is very similar when $\mathbf{u} \varphi$ is a factor of either $x^{r_{1}}$ or $x^{r_{2}}$. Then $\mathbf{u} \varphi=y_{i}^{k}$ for some $k \in\left\{2, \ldots, s_{i}\right\}$. Hence

$$
\mathbf{x}=\underbrace{x^{r_{1}} y_{1}^{s_{1}} \cdots y_{i-1}^{s_{i-1}} y_{i}^{k^{\prime}}}_{\mathbf{e}} \cdot \underbrace{y_{i}^{k}}_{\mathbf{u} \varphi} \cdot \underbrace{y_{i}^{k^{\prime \prime}} y_{i+1}^{s_{i+1}} \cdots y_{n}^{s_{n}} x^{r_{2}}}_{\mathbf{f}}
$$

for some $k^{\prime}, k^{\prime \prime} \geq 0$ such that $k^{\prime}+k+k^{\prime \prime}=s_{i}$. Since the semigroup $L$ satisfies the identity $\mathbf{u} \approx \mathbf{v}$, we have $\operatorname{sim}(\mathbf{u})=\operatorname{sim}(\mathbf{v})$ and non $(\mathbf{u})=$ non $(\mathbf{v})$ by Lemma 1 . It follows that $\mathbf{v} \varphi=y_{i}^{\ell}$ for some $\ell \geq 2$, whence $\mathbf{e}(\mathbf{v} \varphi) \mathbf{f} \in \mathcal{P}_{n}$ in view of (3).

Case 2. $\mathbf{u} \varphi$ belongs to (F2). Then

$$
\mathbf{x}=\underbrace{x^{r_{1}-q_{1}}}_{\mathbf{e}} \cdot \underbrace{x^{q_{1}} y_{1}^{s_{1}} \cdots y_{n}^{s_{n}} x^{q_{2}}}_{\mathbf{u} \varphi} \cdot \underbrace{x^{r_{2}-q_{2}}}_{\mathbf{f}} .
$$

By assumption, $|\operatorname{con}(\mathbf{u})|<n+1=|\operatorname{con}(\mathbf{u} \varphi)|$. Hence there exists some letter $z$ of $\mathbf{u}$ such that the factor $z \varphi$ of $\mathbf{u} \varphi$ contains at least two distinct letters. Therefore the word $z \varphi$ contains one of the following factors: $x y_{1}, y_{1} y_{2}, \ldots, y_{n-1} y_{n}, y_{n} x$. Now one of these is a factor of $\mathbf{v} \varphi$ so that $\mathbf{e}(\mathbf{v} \varphi) \mathbf{f} \notin Q_{n}$. Since $\mathbf{e}(\mathbf{u} \varphi) \mathbf{f} \in \mathcal{P}_{n}$ and the identity $\mathbf{e}(\mathbf{u} \varphi) \mathbf{f} \approx \mathbf{e}(\mathbf{v} \varphi) \mathbf{f}$ is satisfied by the semigroup $L$, we have $\mathbf{e}(\mathbf{v} \varphi) \mathbf{f} \in \mathcal{P}_{n} \cup \mathcal{Q}_{n}$ by Lemma 5. Consequently, $\mathbf{e}(\mathbf{v} \varphi) \mathbf{f} \in \mathcal{P}_{n}$.

\section{Main result}

Lemma 7. For each $n \geq 2$, the semigroup L satisfies the identity $\mathbf{p}_{(n)} \approx \mathbf{q}_{(n)}$ where

$$
\mathbf{p}_{(n)}=x y_{1}^{2} \cdots y_{n}^{2} x \quad \text { and } \quad \mathbf{q}_{(n)}=x y_{n}^{2} \cdots y_{1}^{2} x .
$$

Proof. Let $\varphi$ be any substitution into $L$. It is easy to check that $z L z=\{0\}$ and $z^{2}=0$ for any $z \in\{0, a, b, c\}$. Hence we have $\mathbf{p}_{(n)} \varphi=0=\mathbf{q}_{(n)} \varphi$ whenever $x \varphi \in\{0, a, b, c\}$ or $y_{i} \varphi \in$ $\{0, a, b, c\}$ for some $i$. Therefore it suffices to assume that $x \varphi, y_{1} \varphi, \ldots, y_{n} \varphi \in\{d, e\}$. It is also clear that the condition $y_{1} \varphi=\cdots=y_{n} \varphi$ implies that $\mathbf{p}_{(n)} \varphi=\mathbf{q}_{(n)} \varphi$. Hence further assume that $\left\{y_{1} \varphi, \ldots, y_{n} \varphi\right\}=\{d, e\}$. Then there are two cases depending on how the elements $d$ and $e$ appear in the sequence $\left(y_{1} \varphi, \ldots, y_{n} \varphi\right)$. 
Case 1. Some $e$ in the sequence $\left(y_{1} \varphi, \ldots, y_{n} \varphi\right)$ is sandwiched by two or more occurrences of $d$. Then it is easy to show that $\left(y_{1}^{2} \cdots y_{n}^{2}\right) \varphi=0=\left(y_{n}^{2} \cdots y_{1}^{2}\right) \varphi$, whence $\mathbf{p}_{(n)} \varphi=0=\mathbf{q}_{(n)} \varphi$.

Case 2. Each $e$ in the sequence $\left(y_{1} \varphi, \ldots, y_{n} \varphi\right)$ is not sandwiched by any two occurrences of $d$. Then the sequence $\left(y_{1} \varphi, \ldots, y_{n} \varphi\right)$ is one of the following:

$$
(d, \ldots, d, e, \ldots, e), \quad(e, \ldots, e, d, \ldots, d), \quad(e, \ldots, e, d, \ldots, d, e, \ldots, e) .
$$

Recall that $x \varphi \in\{e, d\}$. Therefore $\left(y_{1} \varphi, \ldots, y_{n} \varphi\right)=(d, \ldots, d, e, \ldots, e)$ implies that

$$
\mathbf{p}_{(n)} \varphi=(x \varphi) \operatorname{de}(x \varphi)=(x \varphi) e d(x \varphi)=\mathbf{q}_{(n)} \varphi,
$$

$\left(y_{1} \varphi, \ldots, y_{n} \varphi\right)=(e, \ldots, e, d, \ldots, d)$ implies that

$$
\mathbf{p}_{(n)} \varphi=(x \varphi) e d(x \varphi)=(x \varphi) \operatorname{de}(x \varphi)=\mathbf{q}_{(n)} \varphi,
$$

and $\left(y_{1} \varphi, \ldots, y_{n} \varphi\right)=(e, \ldots, e, d, \ldots, d, e, \ldots, e)$ implies that

$$
\mathbf{p}_{(n)} \varphi=(x \varphi) e d e(x \varphi)=\mathbf{q}_{(n)} \varphi .
$$

This concludes the proof.

THEOREM 8. The semigroup L is nonfinitely based.

Proof. Working toward a contradiction, suppose that the semigroup $L$ is finitely based. Then there exist a basis $\Sigma$ for $L$ and some fixed integer $n \geq 2$ such that

(†) if $\mathbf{u} \approx \mathbf{v} \in \Sigma$, then $|\operatorname{con}(\mathbf{u})|,|\operatorname{con}(\mathbf{v})| \leq n$.

By Lemma 3, we may further assume that the identities in $\Sigma$ are connected. Now by Lemma 7, the semigroup $L$ satisfies the identity $\mathbf{p}_{(n)} \approx \mathbf{q}_{(n)}$. Therefore we have some deduction sequence

$$
\mathbf{p}_{(n)}=\mathbf{w}_{0} \Rightarrow \mathbf{w}_{1} \Rightarrow \cdots \Rightarrow \mathbf{w}_{m}=\mathbf{q}_{(n)},
$$

where each deduction $\mathbf{w}_{i} \Rightarrow \mathbf{w}_{i+1}$ indicates that there exist words $\mathbf{e}_{i}, \mathbf{f}_{i} \in \mathcal{X}^{*}$, an identity $\mathbf{u}_{i} \approx \mathbf{v}_{i}$ from $\Sigma$, and an endomorphism $\varphi_{i}$ of $\mathcal{X}^{+}$such that

$$
\mathbf{w}_{i}=\mathbf{e}_{i}\left(\mathbf{u}_{i} \varphi_{i}\right) \mathbf{f}_{i} \quad \text { and } \quad \mathbf{w}_{i+1}=\mathbf{e}_{i}\left(\mathbf{v}_{i} \varphi_{i}\right) \mathbf{f}_{i} .
$$

Since $\mathbf{w}_{0} \in \mathcal{P}_{n}$ and the semigroup $L$ satisfies the identities $\mathbf{w}_{0} \approx \mathbf{w}_{1} \approx \cdots \approx \mathbf{w}_{m}$, we have $\mathbf{w}_{1}, \ldots, \mathbf{w}_{m} \in \mathcal{P}_{n} \cup Q_{n}$ by Lemma 5. Suppose that $\mathbf{w}_{k} \in \mathcal{P}_{n}$. Then $\mathbf{w}_{k}=\mathbf{e}_{k}\left(\mathbf{u}_{k} \varphi_{k}\right) \mathbf{f}_{k}$ where $\mathbf{u}_{k} \approx \mathbf{v}_{k} \in \Sigma$. Since the identity $\mathbf{u}_{k} \approx \mathbf{v}_{k}$ is satisfied by $L$ and $\left|\operatorname{con}\left(\mathbf{u}_{k}\right)\right|$, $\left|\operatorname{con}\left(\mathbf{v}_{k}\right)\right| \leq n$ by $(\dagger)$, we have $\mathbf{e}_{k}\left(\mathbf{v}_{k} \varphi_{k}\right) \mathbf{f}_{k} \in \mathcal{P}_{n}$ by Lemma 6 , that is, $\mathbf{w}_{k+1} \in \mathcal{P}_{n}$. Therefore we have just shown by induction that $\mathbf{w}_{0}, \ldots, \mathbf{w}_{m} \in \mathcal{P}_{n}$, which implies the contradiction $\mathbf{q}_{(n)}=\mathbf{w}_{m} \notin Q_{n}$. Consequently, the finite basis $\Sigma$ for the semigroup $L$ does not exist. 


\section{Acknowledgement}

We thank Dr Lee for bringing the semigroup $L$ to our attention and for his help in checking and revising this paper.

\section{References}

[1] S. Burris and H. P. Sankappanavar, A Course in Universal Algebra (Springer, New York, 1981).

[2] A. Distler and T. W. Kelsey, 'The monoids of orders eight, nine \& ten', Ann. Math. Artif. Intell. 56 (2009), 3-21.

[3] E. W. H. Lee, 'Finite basis problem for semigroups of order five or less: generalization and revisitation', Studia Logica, to appear.

[4] E. W. H. Lee and J. R. Li, 'Minimal non-finitely based monoids', Dissertationes Math. (Rozprawy Mat.) 475 (2011).

[5] E. W. H. Lee and M. V. Volkov, 'On the structure of the lattice of combinatorial Rees-Sushkevich varieties', in: Semigroups and Formal Languages (eds. J. M. André et al.) (World Scientific, Singapore, 2007), pp. 164-187.

[6] E. W. H. Lee and M. V. Volkov, 'Limit varieties generated by completely 0-simple semigroups', Internat. J. Algebra. Comput. 21 (2011), 257-294.

[7] G. I. Mashevitskiǔ, 'An example of a finite semigroup without an irreducible basis of identities in the class of completely 0-simple semigroups', Uspekhi Mat. Nauk 38(2) (1983), 211-212 (in Russian); Russian Math. Surveys 38(2) (1983), 192-193 (English translation).

[8] P. Perkins, 'Bases for equational theories of semigroups', J. Algebra 11 (1969), 298-314.

[9] R. J. Plemmons, 'There are 15973 semigroups of order 6', Math. Algorithms 2 (1967), 2-17.

[10] M. V. Sapir, 'Problems of Burnside type and the finite basis property in varieties of semigroups', Izv. Akad. Nauk SSSR Ser. Mat. 51 (1987), 319-340 (in Russian); Math. USSR-Izv. 30 (1988), 295-314 (English translation).

[11] L. N. Shevrin and M. V. Volkov, 'Identities of semigroups', Izv. Vyssh. Uchebn. Zaved. Mat. (11) (1985), 3-47 (in Russian); Soviet Math. (Iz. VUZ) 29(11) (1985), 1-64 (English translation).

[12] A. N. Trahtman, 'Some finite infinitely basable semigroups', Ural. Gos. Univ. Mat. Zap., Issl. Algebra. System, Sverdlovsk 14(2) (1987), 128-131 (in Russian).

[13] A. N. Trahtman, 'Finiteness of identity bases of five-element semigroups', in: Semigroups and their Homomorphisms (ed. E. S. Lyapin) (Ross. Gos. Ped. Univ., Leningrad, 1991), pp. 76-97 (in Russian).

[14] M. V. Volkov, 'The finite basis question for varieties of semigroups', Mat. Zametki 45(3) (1989), 12-23 (in Russian); Math. Notes 45(3) (1989), 187-194 (English translation).

WEN TING ZHANG, Department of Mathematics, Lanzhou University,

Lanzhou, Gansu 730000, PR China

e-mail: zhangwt@1zu.edu.cn

YAN FENG LUO, Department of Mathematics, Lanzhou University, Lanzhou, Gansu 730000, PR China

e-mail: luoyf@lzu.edu.cn 